\title{
Evaluation of three optical flow-based observation models for tracking
}

\author{
M. Lucena, J.M. Fuertes \\ Departamento de Informatica \\ E.P.S. Universidad de Jaen
}

Avda. de Madrid 35, 23071 Jaen, Spain

\{mlucena,jmf\}@ujaen.es

\author{
N. Perez de la Blanca \\ Departamento de Ciencias de la Computacion e I.A. \\ E.T.S.I.I. Universidad de Granada \\ C/ Periodista Saucedo Aranda s/n \\ nicolas@ugr.es
}

\begin{abstract}
In this paper, we study the use of optical flow as a characteristic for tracking. We analyze the behavior of three flowbased observation models for particle filter algorithms, and compare the results with the ones obtained by a well-known, gradient based, observation model. In theory, optical flow could be used directly to displace an object model, but in practice flow estimation techniques lack the necessary accuracy. In view of the fact that probabilistic tracking algorithms enable imprecise or incomplete information to be handled naturally, these models have been used as a natural means of incorporating flow information into the tracking.
\end{abstract}

\section{Introduction}

Probabilistic models applied to tracking enable us to estimate the a posteriori probability distribution, $p(\mathbf{X} \mid \mathbf{Z})$, of the set of valid configurations for the object to be tracked, represented by a vector $\mathbf{X}$, from the set of measurements $\mathbf{Z}$ taken from the images of the sequence. The likelihood in the previous instant is combined with a dynamical model giving rise to the a priori distribution in the current instant, $p(\mathbf{X})$. The relation between these distributions is given by Bayes' Theorem:

$$
p(\mathbf{X} \mid \mathbf{Z}) \propto p(\mathbf{X}) \cdot p(\mathbf{Z} \mid \mathbf{X})
$$

In order to estimate $p(\mathbf{Z} \mid \mathbf{X})$, known as the observation probability, we will define several observation mod$e l s$, based on the optical flow of the sequence. We will also check their validity within a scheme of particle filter tracking, and compare their performance with the model proposed in [1].

\section{Dynamical model}

The tracking task involves localizing, in each frame of a sequence, the object associated to a state vector that characterizes evidence of the presence of a specific configuration of the model in question. Other authors have successfully used characteristics such as the gradient [1] or intensity distributions [10]. The model which represents the dynamical model of the object will provide an a priori distribution of all the possible configurations at the instant $t_{k}, p\left(\mathbf{X}\left(t_{k}\right)\right)$, from the estimated distributions in previous instants of time. In this paper, a second-order dynamical model has been used in which the two previous states of the object model are considered. This is equivalent to taking a first-order dynamical model with a state vector for the instant $t_{k}$ of the form [1]

$$
\mathcal{X}_{t_{k}}=\left[\mathbf{X}_{t_{k-1}}, \mathbf{X}_{t_{k}}\right]^{T}
$$

The integration of the a priori distribution $p(\mathbf{X})$ with the set $\mathbf{Z}$ of the evidences present in each image, in order to obtain the a posteriori distribution $p(\mathbf{X} \mid \mathbf{Z})$, is obtained with Bayes' Theorem. This fusion of information can be performed, if the distributions are Gaussian, by using Kalman's Filter [4]. However, in general, the distributions involved in the process are not Gaussian and multimodal [2]. Sampling methods for modelling this type of distribution [3] have shown themselves to be very useful, and particle filter algorithms $[1,10]$ based on sets of weighted random samples, enable their propagation to be performed effectively.

\section{Observation models}

\subsection{Observation model based on intensity restric- tions}

This model, described more in detail in [7], uses a technique derived from the Lucas-Kanade algorithm[5], and takes advantage of knowledge about the position of the flow discontinuities predicted by the object model. 
Let $\mathbf{x}=f\left(\mathbf{X}_{t_{k}} ; \mathbf{m}\right)$ (where $\mathbf{X}_{t_{k}}$ defines the specific configuration of the object model, and $\mathbf{m}$ is the parameter vector which associates each point within the model with a point on the image plane), a point belonging to the model outline at the instant $t_{k}$. Let $S$ be a neighborhood of $\mathbf{x}$ subdivided in $S_{i}$ and $S_{e}$ (corresponding respectively to the parts of the neighborhood which remain towards the interior and exterior of the outline of the object), and $\mathbf{d}\left(\mathcal{X}_{t_{k}}, \mathbf{m}\right)$ be calculated using the expression:

$$
\mathbf{d}\left(\mathcal{X}_{t_{k}}, \mathbf{m}\right)=f\left(\mathbf{X}_{t_{k}} ; \mathbf{m}\right)-f\left(\mathbf{X}_{t_{k-1}} ; \mathbf{m}\right)
$$

We then consider the optical flow constant in $S_{i}$ and $S_{e}$ respectively, and use the system of equations proposed in [5] to obtain $\mathbf{f}_{S_{x}}=\left(f_{x}, f_{y}\right)$, where $S_{x}$ shall be $S_{i}$ or $S_{e}$ respectively. The temporal derivatives of the image are computed as

$$
I_{t}(\mathbf{x})=I^{(k)}\left(\mathbf{x}+\mathbf{d}\left(\mathcal{X}_{t_{k}}, \mathbf{m}\right)\right)-I^{(k-1)}(\mathbf{x})
$$

In this way, two different flow estimations are obtained, $\mathbf{f}_{S_{i}}\left(\mathcal{X}_{t_{k}}, \mathbf{m}\right)$ and $\mathbf{f}_{S_{e}}\left(\mathcal{X}_{t_{k}}, \mathbf{m}\right)$, corresponding to the inner and outer area of the neighborhood of $\mathbf{x}$, respectively.

The quadratic differences with the expected flow (which in this case equals zero) are equivalent to the squared norm of the estimated flow vectors:

$$
\begin{aligned}
& Z_{S_{i}}\left(\mathcal{X}_{t_{k}}, \mathbf{m}\right)=\left\|\mathbf{f}_{S_{i}}\left(\mathcal{X}_{t_{k}}, \mathbf{m}\right)\right\|^{2} \\
& Z_{S_{e}}\left(\mathcal{X}_{t_{k}}, \mathbf{m}\right)=\left\|\mathbf{f}_{S_{e}}\left(\mathcal{X}_{t_{k}}, \mathbf{m}\right)\right\|^{2}
\end{aligned}
$$

It should be noted that if the point $\mathbf{x}$ is really placed on an optical flow discontinuity, and the flow in $S_{i}$ matches with $\mathbf{d}\left(\mathcal{X}_{t_{k}}, \mathbf{m}\right)$, the value of $Z_{S_{i}}$ must be close to zero and the value of $Z_{S_{e}}$ must be considerably greater. Using the following expression, these values may be combined and a value of $Z\left(\mathcal{X}_{t_{k}}, \mathbf{m}\right)$ may therefore be obtained:

$$
Z\left(\mathcal{X}_{t_{k}}, \mathbf{m}\right)=\frac{Z_{S_{e}}\left(\mathcal{X}_{t_{k}}, \mathbf{m}\right)}{Z_{S_{e}}\left(\mathcal{X}_{t_{k}}, \mathbf{m}\right)+Z_{S_{i}}\left(\mathcal{X}_{t_{k}}, \mathbf{m}\right)}
$$

The value of $Z\left(\mathcal{X}_{t_{k}}, \mathbf{m}\right)$ satisfies the following properties:

- $0 \leq Z\left(\mathcal{X}_{t_{k}}, \mathbf{m}\right) \leq 1$

- If $Z_{S_{e}}\left(\mathcal{X}_{t_{k}}, \mathbf{m}\right) \gg Z_{S_{i}}\left(\mathcal{X}_{t_{k}}, \mathbf{m}\right)$, then $Z\left(\mathcal{X}_{t_{k}}, \mathbf{m}\right) \rightarrow$ 1 , which indicates that the adjustment is much better in $S_{i}$ than it is in $S_{e}$, and therefore the point must be placed exactly in a flow discontinuity, in which the inner area coincides with the displacement predicted by the model.

- If $Z_{S_{e}}\left(\mathcal{X}_{t_{k}}, \mathbf{m}\right) \ll Z_{S_{i}}\left(\mathcal{X}_{t_{k}}, \mathbf{m}\right)$, then $Z\left(\mathcal{X}_{t_{k}}, \mathbf{m}\right) \rightarrow$ 0 . The adjustment is worse in the inner area than it is in the outer area, and therefore the estimated flow does not match the model's prediction.
- If $Z_{S_{e}}\left(\mathcal{X}_{t_{k}}, \mathbf{m}\right)=Z_{S_{i}}\left(\mathcal{X}_{t_{k}}, \mathbf{m}\right)$, then the adjustment is the same in the inner area as it is in the outer area, and therefore the flow adequately matches the displacement predicted by the model, but it is impossible to guarantee that it is placed on a flow discontinuity. In this case, $Z\left(\mathcal{X}_{t_{k}}, \mathbf{m}\right)=1 / 2$.

We will consider that the presence probability of the measurements obtained for the image, since they have been caused by the point of the outline corresponding to the vector $\mathbf{m}$ of the sample in question, defined by the vector $\mathcal{X}_{t_{k}}$, must be proportional to the function $Z\left(\mathcal{X}_{t_{k}}, \mathbf{m}\right)$ computed previously,

$$
p\left(\mathbf{Z} \mid \mathcal{X}_{t_{k}}, \mathbf{m}_{i}\right) \propto Z\left(\mathcal{X}_{t_{k}}, \mathbf{m}_{i}\right)
$$

and that, given the independence between the different points of the outline,

$$
p\left(\mathbf{Z} \mid \mathcal{X}_{t_{k}}\right) \propto \prod_{i} Z\left(\mathcal{X}_{t_{k}}, \mathbf{m}_{i}\right)
$$

with $\mathbf{m}_{i}$ being the vector which identifies the $i-$ nth point on the outline of the model.

\subsection{Observation model based on similarity mea- sures}

If the model prediction about the object is good and the intensity maps corresponding to the neighborhood of each point are superimposed, the inner part of the model must fit better than the outer part [8]. We can therefore use similarity measurements to model the observation probability $p(\mathbf{Z} \mid \mathbf{X})$.

Let $\mathbf{x}=f\left(\mathbf{X}_{t_{k}} ; \mathbf{m}\right)$ be a point belonging to the model outline at the instant $t_{k}$, let $S$ be a neighborhood of $\mathbf{x}$ subdivided in turn into $S_{i}$ and $S_{e}$, let $\mathbf{d}\left(\mathcal{X}_{t_{k}}, \mathbf{m}\right)$ be calculated from expression (1), and let $I^{(k-1)}$ and $I^{(k)}$ be images corresponding to the instants of time $t_{k-1}$ and $t_{k}$. The quadratic errors are therefore computed in the following way:

$$
\begin{aligned}
& Z_{S_{i}}\left(\mathbf{X}_{t_{k}}, \mathbf{m}\right)= \\
& \quad=\sum_{S_{i}} W(\mathbf{x})\left(I^{(k-1)}(\mathbf{x})-I^{(k)}\left(\mathbf{x}-\mathbf{d}\left(\mathcal{X}_{t_{k}}, \mathbf{m}\right)\right)\right)^{2} \\
& Z_{S_{e}}\left(\mathbf{X}_{t_{k}}, \mathbf{m}\right)= \\
& \quad=\sum_{S_{e}} W(\mathbf{x})\left(I^{(k-1)}(\mathbf{x})-I^{(k)}\left(\mathbf{x}-\mathbf{d}\left(\mathcal{X}_{t_{k}}, \mathbf{m}\right)\right)\right)^{2}
\end{aligned}
$$

where $W(\mathbf{x})$ is a weighting function. Two non negative magnitudes are obtained, that may be combined using expression (3), in order to obtain a value of $Z\left(\mathcal{X}_{t_{k}}, \mathbf{m}\right)$. 


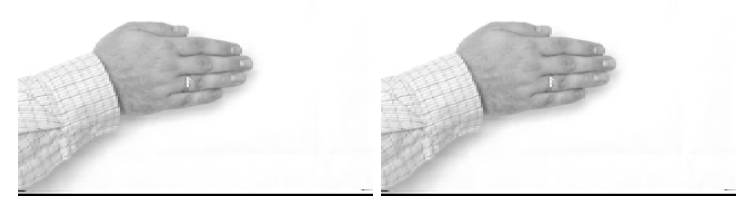

Figure 1. First frame of the two sequences used for the experiments.

\subsection{Observation model based on optical flow}

Let us suppose that an optical flow estimate $\mathbf{v}$ is available for the image $I$ in the instant $t_{k}$. The following error function may be defined [6], with $S \subseteq I$ being an area inside the image:

$$
Z_{S}(\mathbf{v} ; \mathbf{d})=\sum_{(x, y) \in S} W(x, y)\|\mathbf{v}(x, y)-\mathbf{d}(x, y)\|^{2}
$$

where $W(x, y)$ is a weight function and $\mathbf{d}(x, y)$ is derived from equation (1). This measurement will always be nonnegative and will only be equal to zero when the flow vectors are perfectly adjusted to the displacement predicted by the model.

Being $S$ a neighborhood of $f\left(\mathbf{X}_{t_{k-1}} ; \mathbf{m}\right)$, the measurement (7) would be:

$$
Z_{S}\left(\mathcal{X}_{t_{k}}, \mathbf{m}\right)=\sum_{(x, y) \in S} W(x, y)\left\|\mathbf{v}(x, y)-\mathbf{d}\left(\mathcal{X}_{t_{k}}, \mathbf{m}\right)\right\|^{2}
$$

We subdivide $S$ in two areas as before, $S_{i}$ and $S_{e}$, and therefore compute $Z_{S_{i}}\left(\mathcal{X}_{t_{k}}, \mathbf{m}\right)$ and $Z_{S_{e}}\left(\mathcal{X}_{t_{k}}, \mathbf{m}\right)$. Using expression (3), we compute $Z\left(\mathcal{X}_{t_{k}}, \mathbf{m}_{i}\right)$.

\section{Experiments}

We have used two image sequences, with a length of 10 seconds, at 25 frames per second, $320 \times 240$ pixels, and 8 bits per band and pixel. These sequences (figure 1) correspond to the movement of a hand over a background with and without clutter. Results can be downloaded from http://wwwdi.ujaen.es/ mlucena/invest.html.

In order to model the hand, we have used two splinebased contour models. For the first, 20 points/normals are selected, and 18 for the second. The second-order dynamical model for each sequence has been learnt using the multidimensional algorithm proposed in [1].

\begin{tabular}{|l|l|}
\hline Model & Parameters \\
\hline Blake & $\begin{array}{l}\text { 200 samples, } \sigma=3, \\
\text { First sequence: } \alpha=0.025 \\
\text { Second sequence: } \alpha=0.055\end{array}$ \\
\hline Intensity Restric. & 200 samples, 7x7 Neigh. \\
\hline Similarity & 200 samples, 5x5 Neigh. \\
\hline Optical Flow & $\begin{array}{l}200 \text { samples, Proesmans algo- } \\
\text { rithm } \\
W(x, y)=\|\| \nabla I(x, y)\|\|\end{array}$ \\
\hline
\end{tabular}

Table 1. Parameters used for the experiments.

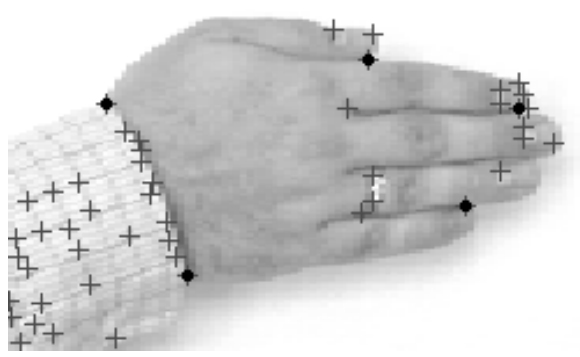

Figure 2. Set of points selected from the first sequence.

We have used the CONDENSATION algorithm with the three proposed models and the contour-normals model (Blake) proposed in [1]. The parameters used for each model and sequence are depicted in table 1 . In order to estimate the optical flow for using with the last observation model, we have used the algoritm proposed in [9]. The weighted average of the distribution obtained is used as the estimated position of the tracked object.

In order to measure the performance of the proposed models, we have proceeded as follows. Given the initial frame of each sequence, we have used the Harris operator to obtain a number of points, and manually selected five points placed on the contour of the object of interest (figure 2 ). We have then located the corresponding points for the entire sequence. When the tracking process is finished, we compute the mean euclidean distance between each point and the position estimated by the tracker for that point. The results obtained are in figure 3.

\section{Discussion and conclusions}

The observation model based on contour normals presents better results for the first sequence, but for the sec- 


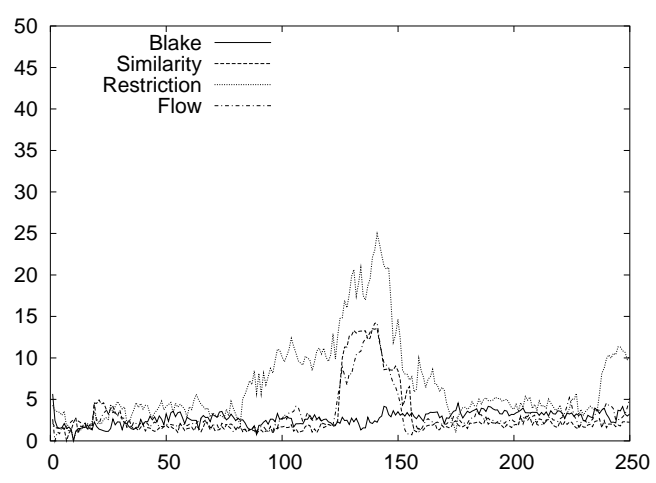

First Sequence

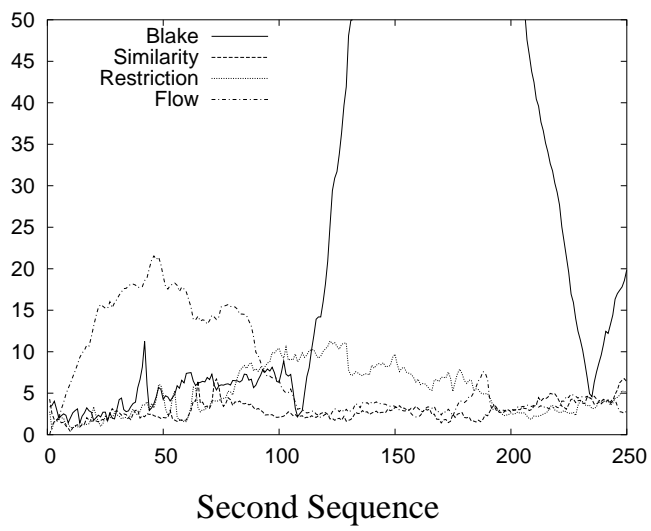

Figure 3. Results.

ond loses the object in a significant number of frames. This is due to the presence of a significant number of edges outside the object, giving rise to a number of local maxima in the observation model.

The intensity restrictions based observation model performs slightly better for the second sequence than for the first. This is due to the absence of texture outside the object, that dificults the estimation of the optical flow. We can see that the model is distracted in the middle part of the sequence. This is caused by an ambiguity caused by the hand's shadow, making impossible to separate it from the hand attending only to the optical flow. This phenomenon also occurs, although in less degree, with the other two observation models.

The performance of the similarity-based observation model is very good for the two sequences. This demonstrates that the presence of clutter is not a problem for this model, in opposition to the results obtained with the Blake's model.

The optical-flow based observation model gives better results for the first sequence. At the beginning of the second sequence, there is mainly horizontal displacement of the object, and the absence of optical flow discontinuities in the arm-hand joint causes some dificulties for the model in order to detect such movements. Nevertheless, the model doesn't loses completely the object.

The experiments presented here suggest that the optical flow is a valid characteristic in order to perform tracking tasks, and can give better results than gradient based models in presence of clutter. Of the three presented observation models, the one based on similarity measures gives the best results, although there are inherent limitations to those models, inherited from the optical flow estimation problem itself.

\section{Acknowledgement}

This work has been financed by grant TIC-2001-3316 from the Spanish Ministry of Science and Technology.

\section{References}

[1] A. Blake and M. Isard. Active Contours. Springer, 1998.

[2] J. Deutscher, A. Blake, B. North, and B. Bascle. Tracking through singularities and discontinuities by random sampling. In Proceedings of International Conference on Computer Vision, volume 2, pages 1144-1149, 1999.

[3] A. Gelfand and A. Smith. Sampling-based approaches to computing marginal densities. Journal of the American Statistical Association, 85(410):398-409, 1990.

[4] R. Kalman. A new approach to linear filtering and prediction problems. Transactions of the ASME-Journal of Basic Engineering, 82(Series D):35-45, 1960.

[5] B. Lucas and T. Kanade. An iterative image registration technique with an application to stereo vision. In Proceedings of DARPA IU Workshop, pages 121-130, 1981.

[6] M. Lucena, J. Fuertes, N. Perez de la Blanca, and A. Garrido. An optical flow probabilistic observation model for tracking. In Proceedings of IEEE International Conference on Image Processing, volume 3, pages 957-960, 2003.

[7] M. Lucena, J. Fuertes, N. Perez de la Blanca, and A. Garrido. Using optical flow as evidende for probabilistic tracking. Lecture Notes in Computer Science, (2749):1045-1049, 2003.

[8] M. Lucena, J. Fuertes, N. Perez de la Blanca, A. Garrido, and J. Gomez. Optical flow-based probabilistic tracking. In Proceedings of VII International Symposium on Signal Processing and its Applcations, volume 2, pages 219-222, 2003.

[9] M. Proesmans, L. Van Gool, E. Pauwels, and A. Oosterlinck. Determination of optical flow and its discontinuities using non-linear diffusion. In Proceedings of 3rd European Conference on Computer Vision, volume 2, pages 295-304, 1994.

[10] J. Sullivan, A. Blake, M. Isard, and J. MacCormick. Bayesian object localisation in images. International Journal of Computer Vision, 44(2):111-135, 2001. 